\section{Tendência temporal da prevalência de obesidade mórbida na população adulta brasileira entre os anos de 2006 e 2017}

\author{
Time trends in morbid obesity prevalence in the \\ Brazilian adult population from 2006 to 2017
}

Deborah Carvalho Malta 1

Alanna Gomes da Silva 1

Luis Antônio Batista Tonaco 1

Maria Imaculada de Fátima Freitas 1

Gustavo Velasquez-Melendez 1

\title{
Resumo
}

Para a avaliação da tendência temporal da prevalência de obesidade mórbida na população adulta das capitais brasileiras entre os anos 2006 e 2017, foi realizado estudo transversal com dados da Vigilância de Fatores de Risco e Proteção para Doenças Crônicas por Inquérito Telefônico (Vigitel), analisados por regressão linear simples. Resultados evidenciaram tendência de aumento da prevalência de obesidade mórbida no Brasil. As mulheres apresentaram maiores prevalências (1,3\%, em 2006, e 1,9\%, em 2017) ao serem comparadas com os homens (0,9\% e 1,4\%). Na faixa etária entre 25 a 44 anos, a tendência foi de crescimento de 0,9\% para 2,1\% ( $p<0,001)$. Em todos os níveis de escolaridade e regiões do Brasil, houve aumento da obesidade mórbida. As capitais que apresentaram tendência de aumento no sexo masculino foram: Campo Grande, Curitiba, Fortaleza, Macapá, Manaus, Palmas, Porto Velho, Rio Branco e Teresina. Em relação ao sexo feminino: Belo Horizonte, Campo Grande, Rio de Janeiro e Teresina. O crescimento da obesidade mórbida no país constitui um alerta para a urgente necessidade de adotar medidas para detê-la, como a regulação de alimentos ultraprocessados e ações de educação para a saúde para toda a população.

Doenças Não Transmissíveis; Obesidade Mórbida; Inquéritos Epidemiológicos; Promoção da Saúde

\author{
Correspondência \\ D. C. Malta \\ Escola de Enfermagem, Universidade Federal de Minas Gerais. \\ Av. Prof. Alfredo Balena 190, Belo Horizonte, MG \\ 30130-100, Brasil. \\ dcmalta@uol.com.br \\ 1 Escola de Enfermagem, Universidade Federal de Minas Gerais, \\ Belo Horizonte, Brasil.
}




\section{Introdução}

A epidemia de obesidade é um fenômeno mundial e sua prevalência triplicou desde 1975 1. Em 2015, no Reino Unido, o número de adultos com obesidade mórbida foi de 1,9 milhão e estima-se um aumento para 4 milhões em 2035 2. Nos Estados Unidos, também houve aumento de 5,7\%, em 2008, para $7,7 \%$, em $2016{ }^{3}$. Além disso, o índice de massa corporal (IMC) tem se elevado em cerca de $0,4 \mathrm{~kg} / \mathrm{m}^{2}$ por década em todo o mundo 4 .

A obesidade mórbida ou obesidade grave grau III se define pelo IMC $\geq 40 \mathrm{~kg} / \mathrm{m}^{25,6}$ e é responsável pelo aumento da morbidade, mortalidade e de custos dos cuidados de saúde 7 . Em adultos, está relacionada às doenças crônicas não transmissíveis (DCNT), como complicações metabólicas, doenças cardíacas, cerebrovasculares, embolia pulmonar, diabetes tipo 2, dislipidemias, distúrbios musculoesqueléticos e alguns tipos de câncer ${ }^{8}$.

As causas são multifatoriais, incluindo alimentação inadequada, aumento do consumo de alimentos processados de alta densidade calórica, gasto energético diminuído devido ao sedentarismo, além de fatores genéticos, metabólicos, endócrinos, psicológicos, sociais e culturais 9,10.

Deter o crescimento da obesidade constitui uma das metas do Plano Global de DCNT (20132020) 11 e do Plano de Enfrentamento de DCNT no Brasil (2011-2022) 12. O sistema de Vigilância de Fatores de Risco e Proteção para Doenças Crônicas por Inquérito Telefônico (Vigitel) é uma ferramenta imprescindível para o monitoramento da frequência e distribuição dos principais determinantes das DCNT e dos fatores de risco.

Neste estudo, analisou-se a tendência temporal da prevalência de obesidade mórbida na população adulta das capitais brasileiras, entre os anos de 2006 e 2017.

\section{Métodos}

Trata-se de estudo transversal descritivo sobre a tendência temporal da prevalência de obesidade mórbida entre os anos de 2006 e 2017, a partir de informações do Vigitel.

O Vigitel é um estudo de base populacional, que avalia, anualmente, a população adulta, com idade igual ou maior que 18 anos, residente nas capitais dos 26 estados brasileiros e no Distrito Federal, por meio de entrevistas telefônicas ${ }^{13}$. As perguntas do questionário do Vigitel contemplam as características demográficas e socioeconômicas dos indivíduos, incluindo o peso e a altura 13 .

As prevalências analisadas neste estudo foram calculadas com base no IMC, sendo o peso e a altura informações autorreferidas. Foi realizada a imputação de dados faltantes de peso e altura em todos os anos da série. O método de imputação utilizado foi o hot deck, no qual é identificada a associação entre as variáveis idade, sexo, escolaridade e raça/cor da pele e a não resposta para peso e altura. Todas as estimativas do Vigitel são ponderadas para que sejam representativas do conjunto da população adulta de cada cidade. O cálculo do peso pós-estratificação é feito pelo método rake e considera as variáveis sexo, faixa etária e nível de escolaridade. Outros detalhes da metodologia empregada pelo sistema são fornecidos em publicações do Vigitel 13,14.

Para identificar a existência de tendência linear do indicador, foram utilizados modelos de regressão linear como variável dependente a prevalência da obesidade mórbida e como variável explicativa o ano do levantamento. $\mathrm{O}$ valor do coeficiente angular desses modelos expressa a variação média anual (aumento ou redução) da prevalência da obesidade mórbida. Considerou-se a existência de tendência linear significativa quando o coeficiente angular do modelo mostrou-se diferente de zero para um valor p inferior a 0,05. Como medida de adequação do modelo foi utilizada a análise de resíduo e o R2 que indica o percentual de explicação da variabilidade dos valores da variável dependente.

Foram analisados tendências por sexo, faixa etária, escolaridade e para as capitais brasileiras e o total das capitais. Para o processamento dos dados e análises estatísticas, foi utilizado o programa Stata, versão 14 (https://www.stata.com).

O projeto Vigitel foi aprovado pela Comissão Nacional de Ética em Pesquisa para Seres Humanos do Ministério da Saúde (parecer no 2.100.213 - CAAE: 65610017.1.0000.0008). O consentimento livre e esclarecido foi obtido oralmente no momento do contato telefônico com os entrevistados. 


\section{Resultados}

Houve tendência de aumento da prevalência da obesidade mórbida no Brasil $(\mathrm{p}<0,001)$. As mulheres apresentaram maior prevalência (1,3\%, em 2006, e 1,9\%, em 2017 - inclinação 0,080) ao serem comparadas aos homens $(0,9 \%$ e $1,4 \%$ - inclinação 0,060$)$. Não houve tendência significativa da prevalência de obesidade mórbida nas faixas etárias de 18 a 24 anos $(\mathrm{p}=0,072), 45$ a $54(\mathrm{p}=0,243), 55$ a 64 $(\mathrm{p}=0,239) \mathrm{e} \geq 65$ anos $(\mathrm{p}=0,450)$. Porém, na faixa etária entre $25 \mathrm{a} 44$ anos, a tendência foi de aumento $(\mathrm{p}<0,001)$. Para todos os níveis de escolaridade, houve aumento da prevalência de obesidade mórbida: os indivíduos com 0 a 8 anos de estudo apresentaram maiores prevalências durante todo período analisado $(1,8 \%$, em 2006, e 2,8\%, em 2017 - inclinação 0,120$)$ ao serem comparados com aqueles com 9 a 11 anos $(0,5 \%$, em 2006, e 1,4\%, em 2017 - inclinação 0,090) e com 12 ou mais anos de estudo (0,5\%, em 2006, e 0,9\%, em 2017 - inclinação 0,043). Em todas as regiões do Brasil, a tendência foi de aumento, com maiores prevalências nas regiões Norte e Sudeste (Tabela 1).

As capitais que apresentaram variações significativas com tendência de aumento $(p<0,05)$ no sexo masculino foram: Campo Grande, Curitiba, Fortaleza, Macapá, Manaus, Palmas, Porto Velho, Rio Branco e Teresina. As maiores prevalências de obesidade mórbida em 2017 foram em Rio Branco $(3,4 \%)$ e Porto Velho $(3,3 \%)$ (Tabela 2$)$.

Houve aumento significativo da prevalência de obesidade mórbida no sexo feminino, nas seguintes capitais: Belo Horizonte, Campo Grande, Rio de Janeiro e Teresina, com prevalências mais elevadas no ano de 2017 em Teresina (2,5\%) e Campo Grande (2,4\%) (Tabela 3).

\section{Tabela 1}

Tendência de obesidade mórbida na população das capitais brasileiras, segundo características sociodemográficas. Vigilância de Fatores de Risco e Proteção para Doenças Crônicas por Inquérito Telefônico (Vigitel), 2006 a 2017.

\begin{tabular}{|c|c|c|c|c|c|c|c|c|c|c|c|c|c|c|c|}
\hline Variáveis & 2006 & 2007 & 2008 & 2009 & 2010 & 2011 & 2012 & 2013 & 2014 & 2015 & 2016 & 2017 & Valor de $p$ & Inclinação & $\mathbf{R}^{2}$ \\
\hline Total & 1,1 & 1,0 & 0,9 & 1,2 & 1,2 & 1,2 & 1,5 & 1,5 & 1,6 & 1,7 & 1,6 & 1,7 & $<0,001$ & 0,073 & 0,869 \\
\hline \multicolumn{16}{|l|}{ Sexo } \\
\hline Masculino & 0,9 & 0,8 & 0,5 & 0,7 & 0,8 & 0,8 & 1,0 & 1,2 & 1,2 & 1,1 & 1,2 & 1,4 & $<0,001$ & 0,060 & 0,692 \\
\hline Feminino & 1,3 & 1,2 & 1,3 & 1,6 & 1,6 & 1,4 & 2,0 & 1,8 & 1,9 & 2,2 & 2,0 & 1,9 & $<0,001$ & 0,080 & 0,759 \\
\hline \multicolumn{16}{|l|}{ Idade (anos) } \\
\hline $18-24$ & 0,3 & 0,5 & 0,6 & 0,5 & 0,3 & 0,3 & 0,3 & 0,3 & 0,6 & 0,9 & 0,9 & 0,6 & 0,072 & 0,033 & 0,287 \\
\hline $25-34$ & 0,9 & 0,9 & 0,7 & 1,0 & 0,9 & 1,0 & 1,4 & 1,1 & 1,8 & 1,8 & 1,6 & 1,5 & $<0,001$ & 0,090 & 0,708 \\
\hline $35-44$ & 0,9 & 1,0 & 1,1 & 1,2 & 1,3 & 1,2 & 1,6 & 1,8 & 1,8 & 2,2 & 2,4 & 2,1 & $<0,001$ & 0,134 & 0,917 \\
\hline $45-54$ & 1,7 & 1,7 & 0,9 & 1,7 & 1,9 & 1,9 & 1,8 & 2,1 & 1,4 & 1,9 & 1,6 & 2,1 & 0,243 & 0,033 & 0,133 \\
\hline $55-64$ & 1,6 & 1,2 & 1,6 & 1,4 & 1,9 & 1,2 & 2,7 & 2,4 & 1,9 & 1,9 & 1,8 & 1,6 & 0,239 & 0,045 & 0,135 \\
\hline$\geq 65$ & 1,9 & 1,0 & 1,1 & 1,6 & 1,6 & 1,3 & 1,8 & 1,9 & 2,0 & 1,2 & 1,3 & 1,9 & 0,450 & 0,024 & 0,058 \\
\hline \multicolumn{16}{|c|}{ Escolaridade (anos) } \\
\hline $0-8$ & 1,8 & 1,6 & 1,2 & 1,8 & 2,0 & 1,5 & 2,1 & 2,4 & 2,6 & 2,3 & 2,7 & 2,8 & $<0,001$ & 0,120 & 0,731 \\
\hline $9-11$ & 0,5 & 0,5 & 0,8 & 0,9 & 0,7 & 0,9 & 1,4 & 0,9 & 1,3 & 1,6 & 1,3 & 1,4 & $<0,001$ & 0,090 & 0,767 \\
\hline$\geq 12$ & 0,5 & 0,6 & 0,6 & 0,6 & 0,7 & 0,9 & 0,9 & 1,2 & 0,6 & 1,0 & 1,0 & 0,9 & 0,010 & 0,043 & 0,499 \\
\hline \multicolumn{16}{|l|}{ Região } \\
\hline Norte & 1,3 & 1,1 & 1,2 & 1,4 & 1,4 & 1,3 & 1,8 & 1,3 & 1,7 & 2,3 & 1,6 & 1,8 & 0,007 & 0,068 & 0,523 \\
\hline Nordeste & 0,9 & 1,2 & 1,2 & 1,4 & 1,5 & 1,3 & 1,3 & 1,5 & 1,8 & 1,4 & 1,8 & 1,7 & $<0,001$ & 0,062 & 0,697 \\
\hline Centro-oeste & 0,6 & 0,8 & 0,9 & 0,8 & 0,8 & 1,0 & 1,3 & 1,2 & 2,0 & 1,0 & 1,3 & 1,1 & 0,028 & 0,064 & 0,394 \\
\hline Sudeste & 1,3 & 1,0 & 0,7 & 1,2 & 1,2 & 1,1 & 1,7 & 1,7 & 1,3 & 2,0 & 1,7 & 1,8 & 0,003 & 0,082 & 0,592 \\
\hline Sul & 1,1 & 0,8 & 1,2 & 0,9 & 1,1 & 0,9 & 1,3 & 1,3 & 1,5 & 1,5 & 1,4 & 1,5 & 0,002 & 0,056 & 0,641 \\
\hline
\end{tabular}


Tabela 2

Tendência da prevalência de obesidade mórbida na população do sexo masculino das capitais brasileiras. Vigilância de Fatores de Risco e Proteção para Doenças Crônicas por Inquérito Telefônico (Vigitel), 2006 a 2017.

\begin{tabular}{|c|c|c|c|c|c|c|c|c|c|c|c|c|c|c|c|}
\hline Capitais & 2006 & 2007 & 2008 & 2009 & 2010 & 2011 & 2012 & 2013 & 2014 & 2015 & 2016 & 2017 & Valor de p & Inclinação & $\mathbf{R}^{2}$ \\
\hline Aracaju & 1,3 & 0,5 & 0,4 & 1,1 & 1,9 & 0,9 & 2,2 & 0,9 & 1,2 & 1,1 & 2,1 & 2,8 & 0,040 & 0,121 & 0,356 \\
\hline Belém & 1,4 & 1,1 & 0,5 & 0,8 & 1,1 & 0,4 & 0,7 & 0,5 & 0,9 & 1,5 & 0,9 & 0,8 & 0,787 & $-0,008$ & 0,007 \\
\hline Belo Horizonte & 0,4 & 0,9 & 0,5 & 1,2 & 0,6 & 0,6 & 0,7 & 1,1 & 1,1 & 0,5 & 0,3 & 1,6 & 0,364 & 0,031 & 0,083 \\
\hline Boa Vista & 0,6 & 0,9 & 1,3 & 0,6 & 0,7 & 0,4 & 0,9 & 0,7 & 2,5 & 0,8 & 0,8 & 1,3 & 0,338 & 0,047 & 0,091 \\
\hline Campo Grande & 0,7 & 0,4 & 0,5 & 0,5 & 1,4 & 0,6 & 0,8 & 0,9 & 1,5 & 1,8 & 2,0 & 2,5 & $<0,001$ & 0,164 & 0,736 \\
\hline Cuiabá & 0,6 & 0,6 & 0,7 & 0,7 & 1,1 & 1,3 & 1,0 & 2,1 & 2,4 & 0,5 & 0,4 & 1,2 & 0,358 & 0,051 & 0,084 \\
\hline Curitiba & 0,9 & 0,4 & 0,6 & 0,3 & 0,9 & 0,5 & 1,4 & 1,5 & 1,5 & 0,7 & 1,8 & 1,2 & 0,024 & 0,088 & 0,414 \\
\hline Florianópolis & 0,2 & 0,9 & 0,9 & 0,4 & 0,6 & 0,7 & 1,6 & 0,6 & 2,8 & 1,5 & 1,3 & 0,7 & 0,121 & 0,092 & 0,219 \\
\hline Fortaleza & 0,6 & 0,3 & 0,9 & 0,4 & 1,5 & 0,4 & 0,5 & 1,1 & 2,2 & 1,2 & 1,3 & 1,6 & 0,024 & 0,105 & 0,411 \\
\hline Goiânia & 0,4 & 0,6 & 0,4 & 0,7 & 0,6 & 0,5 & 1,3 & 0,6 & 0,2 & 0,3 & 1,9 & 0,6 & 0,333 & 0,040 & 0,093 \\
\hline João Pessoa & 1,1 & 0,9 & 1,6 & 0,6 & 0,8 & 1,4 & 1,1 & 1,0 & 1,1 & 0,8 & 2,6 & 0,8 & 0,497 & 0,032 & 0,047 \\
\hline Macapá & 0,5 & 0,8 & 0,4 & 0,9 & 0,7 & 0,3 & 0,9 & 1,4 & 1,1 & 2,2 & 1,7 & 5,1 & 0,008 & 0,262 & 0,522 \\
\hline Maceió & 0,3 & 0,8 & 1,2 & 2,3 & 0,8 & 1,1 & 0,6 & 0,8 & 0,5 & 1,3 & 1,5 & 1,7 & 0,365 & 0,045 & 0,082 \\
\hline Manaus & 0,8 & 1,0 & 0,6 & 0,7 & 1,3 & 1,3 & 0,7 & 1,2 & 1,6 & 2,4 & 1,7 & 1,1 & 0,028 & 0,090 & 0,395 \\
\hline Natal & 1,4 & 0,9 & 0,5 & 0,8 & 0,8 & 1,9 & 2,0 & 1,4 & 1,8 & 1,6 & 1,4 & 1,4 & 0,096 & 0,067 & 0,252 \\
\hline Palmas & 0,5 & 0,2 & 0,1 & 0,9 & 0,8 & 0,4 & 1,3 & 0,9 & 1,0 & 0,6 & 1,2 & 1,2 & 0,013 & 0,077 & 0,479 \\
\hline Porto Alegre & 1,2 & 0,6 & 0,9 & 0,3 & 1,2 & 0,8 & 0,5 & 2,3 & 1,2 & 1,7 & 1,1 & 1,0 & 0,251 & 0,054 & 0,129 \\
\hline Porto Velho & 0,2 & 0,9 & 2,0 & 0,9 & 0,8 & 1,0 & 1,2 & 1,8 & 1,7 & 1,2 & 1,7 & 3,3 & 0,014 & 0,150 & 0,469 \\
\hline Recife & 0,6 & 0,3 & 0,3 & 2,1 & 0,8 & 1,1 & 0,5 & 1,4 & 0,5 & 1,0 & 1,1 & 2,2 & 0,141 & 0,080 & 0,203 \\
\hline Rio Branco & 0,3 & 1,4 & 1,7 & 0,6 & 1,0 & 0,9 & 1,2 & 0,8 & 2,8 & 1,7 & 2,4 & 3,4 & 0,008 & 0,188 & 0,525 \\
\hline Rio de Janeiro & 0,9 & 0,9 & 0,4 & 1,0 & 1,0 & 1,2 & 1,2 & 0,6 & 1,0 & 0,4 & 1,7 & 1,3 & 0,265 & 0,036 & 0,122 \\
\hline Salvador & 0,4 & 1,6 & 0,4 & 0,9 & 0,9 & 0,8 & 0,6 & 0,5 & 0,5 & 0,6 & 1,2 & 1,5 & 0,537 & 0,023 & 0,039 \\
\hline São Luís & 0,9 & 0,0 & 0,1 & 0,8 & 0,5 & 0,7 & 0,9 & 0,5 & 0,2 & 0,6 & 1,0 & 0,7 & 0,363 & 0,026 & 0,083 \\
\hline São Paulo & 1,4 & 0,9 & 0,3 & 0,2 & 0,5 & 0,9 & 1,4 & 1,8 & 1,1 & 1,6 & 0,8 & 1,7 & 0,119 & 0,071 & 0,225 \\
\hline Teresina & 0,2 & 0,4 & 0,4 & 0,3 & 0,4 & 1,0 & 0,2 & 0,9 & 0,4 & 0,7 & 0,7 & 0,9 & 0,036 & 0,048 & 0,369 \\
\hline Vitória & 0,3 & 0,6 & 0,1 & 0,6 & 1,1 & 1,0 & 0,5 & 0,7 & 2,2 & 0,6 & 0,3 & 1,5 & 0,156 & 0,071 & 0,190 \\
\hline Brasília & 0,2 & 0,2 & 0,7 & 0,3 & 0,5 & 0,1 & 0,6 & 0,6 & 1,4 & 0,5 & 0,9 & 0,1 & 0,284 & 0,035 & 0,113 \\
\hline
\end{tabular}

\section{Discussão}

A tendência de aumento da prevalência de obesidade mórbida ocorreu para ambos os sexos da população brasileira, nos indivíduos na faixa etária de 25 a 44 anos, em moradores de todas as regiões e em todos os níveis de escolaridade.

O aumento dessa prevalência ocorre não apenas no Brasil, mas em outros países e tem sido atribuído à alimentação inadequada e aos baixos níveis de atividade física. Outros estudos com dados do Vigitel mostraram alta prevalência do consumo regular de refrigerantes (28\%) e de carne com gordura (46\%), baixa proporção de atividade física no lazer (15\%) e do consumo de frutas e hortaliças (18\%) pela população adulta das capitais brasileiras, considerados determinantes para o aumento da obesidade 15 .

Políticas governamentais para incremento de consumo de alimentos saudáveis foram implementadas nos últimos anos, por meio do Ministério da Saúde, tais como, obrigatoriedade de rotulagem dos alimentos, acordos com a indústria para a eliminação das gorduras trans e redução de sal nos alimentos processados e ultraprocessados, além de definição e respectivas recomendações publicadas no Guia Alimentar para a População Brasielira de 2014 16. Contudo, a alta frequência da substituição das refeições por lanches e alta prevalência do consumo de doces e sal, ainda existentes, reforçam a importância de avançar em outras ações e políticas ${ }^{17}$. Alguns exemplos podem ser citados, como a implementação de medidas para proibir propagandas de alimentação destinadas às crianças, o aumento de 
Tabela 3

Tendência da prevalência de obesidade mórbida na população do sexo feminino das capitais brasileiras. Vigilância de Fatores de Risco e Proteção para Doenças Crônicas por Inquérito Telefônico (Vigitel), 2006 a 2017.

\begin{tabular}{|c|c|c|c|c|c|c|c|c|c|c|c|c|c|c|c|}
\hline Capitais & 2006 & 2007 & 2008 & 2009 & 2010 & 2011 & 2012 & 2013 & 2014 & 2015 & 2016 & 2017 & Valor de $p$ & Inclinação & $\mathbf{R}^{2}$ \\
\hline Aracaju & 1,4 & 2,2 & 1,4 & 1,6 & 2,1 & 1,8 & 2,4 & 1,8 & 1,0 & 1,9 & 2,6 & 1,5 & 0,679 & 0,017 & 0,017 \\
\hline Belém & 1,7 & 0,9 & 1,4 & 1,9 & 0,9 & 1,3 & 2,8 & 1,6 & 2,2 & 2,4 & 2,3 & 1,3 & 0,172 & $-0,071$ & 0,178 \\
\hline Belo Horizonte & 1,1 & 1,0 & 1,3 & 1,6 & 1,5 & 1,2 & 1,6 & 1,5 & 1,4 & 2,2 & 2,0 & 2,1 & $<0,001$ & 0,090 & 0,700 \\
\hline Boa Vista & 1,1 & 1,2 & 1,6 & 1,1 & 1,7 & 0,8 & 1,4 & 1,3 & 1,2 & 1,3 & 0,3 & 1,9 & 0,889 & 0,005 & 0,002 \\
\hline Campo Grande & 1,0 & 1,8 & 1,3 & 2,1 & 1,7 & 2 & 2,1 & 1,4 & 2,6 & 2,9 & 2,1 & 2,4 & 0,009 & 0,108 & 0,507 \\
\hline Cuiabá & 1,7 & 1,6 & 0,8 & 1,5 & 1,9 & 2,3 & 2,2 & 2,7 & 3,3 & 1,5 & 2,0 & 2,0 & 0,136 & 0,081 & 0,207 \\
\hline Curitiba & 1,1 & 1,1 & 1,6 & 0,9 & 1,0 & 1,2 & 1,6 & 0,8 & 1,4 & 1,6 & 0,8 & 1,8 & 0,406 & 0,026 & 0,070 \\
\hline Florianópolis & 0,9 & 0,9 & 0,7 & 1,3 & 1,0 & 0,9 & 1,7 & 1,0 & 1,0 & 1,7 & 0,5 & 0,8 & 0,865 & 0,006 & 0,003 \\
\hline Fortaleza & 0,9 & 1,4 & 1,6 & 1,6 & 1,0 & 1,9 & 0,8 & 1,8 & 3,5 & 1,5 & 2,3 & 1,7 & 0,121 & 0,094 & 0,222 \\
\hline Goiânia & 1,0 & 1,0 & 0,8 & 0,9 & 1,1 & 0,8 & 0,8 & 1,2 & 1,6 & 1,2 & 1,4 & 1,1 & 0,052 & 0,040 & 0,327 \\
\hline João Pessoa & 1,1 & 0,7 & 2,2 & 2,0 & 1,5 & 1,5 & 1,4 & 1,9 & 1,0 & 1,4 & 1,5 & 2,3 & 0,372 & 0,038 & 0,080 \\
\hline Macapá & 1,4 & 2,1 & 2,2 & 2,3 & 4,8 & 2,6 & 2,1 & 1,5 & 2,9 & 2,3 & 2,1 & 1,7 & 0,881 & 0,012 & 0,002 \\
\hline Maceió & 0,6 & 1,7 & 3,1 & 3,2 & 1,3 & 2,0 & 1,2 & 3,2 & 1,9 & 2,6 & 3,0 & 2,5 & 0,203 & 0,096 & 0,156 \\
\hline Manaus & 2,0 & 1,3 & 1,5 & 2,2 & 1,4 & 1,9 & 3,0 & 1,8 & 1,9 & 3,9 & 1,5 & 2,2 & 0,241 & 0,076 & 0,134 \\
\hline Natal & 1,3 & 2,0 & 0,9 & 2,0 & 1,9 & 2,0 & 1,8 & 1,3 & 2,2 & 2,4 & 2,2 & 1,7 & 0,142 & 0,055 & 0,202 \\
\hline Palmas & 0,9 & 0,9 & 1,2 & 0,5 & 1,1 & 1,4 & 2,1 & 0,8 & 1,5 & 0,4 & 1,5 & 1,1 & 0,571 & 0,024 & 0,033 \\
\hline Porto Alegre & 1,4 & 0,9 & 1,9 & 2,2 & 1,5 & 1,1 & 1,3 & 0,9 & 1,5 & 2,0 & 2,4 & 2,2 & 0,159 & 0,063 & 0,187 \\
\hline Porto Velho & 1,1 & 1,9 & 1,2 & 2,3 & 2,9 & 3,3 & 2,2 & 1,6 & 1,5 & 3,0 & 1,4 & 1,6 & 0,808 & 0,016 & 0,006 \\
\hline Recife & 1,3 & 2,0 & 1,8 & 1,2 & 2,5 & 1,4 & 2,7 & 2,1 & 2,5 & 1,8 & 2,6 & 1,8 & 0,168 & 0,061 & 0,180 \\
\hline Rio Branco & 1,4 & 1,5 & 3,6 & 2,5 & 3,0 & 2,6 & 1,8 & 1,3 & 1,0 & 3,3 & 2,4 & 2,1 & 0,989 & 0,001 & 0,000 \\
\hline Rio de Janeiro & 1,0 & 1,2 & 1,8 & 1,7 & 1,9 & 1,4 & 2,4 & 3,1 & 2,3 & 3,0 & 2,9 & 2,1 & 0,003 & 0,152 & 0,613 \\
\hline Salvador & 1,2 & 1,7 & 2,3 & 2,0 & 2,7 & 1,5 & 2,0 & 2,3 & 2,6 & 1,5 & 2,6 & 2,1 & 0,222 & 0,051 & 0,144 \\
\hline São Luís & 0,9 & 1,9 & 0,4 & 1,3 & 1,4 & 1,6 & 1,5 & 1,5 & 1,6 & 0,9 & 0,7 & 1,2 & 0,837 & $-0,008$ & 0,004 \\
\hline São Paulo & 1,7 & 1,0 & 0,3 & 1,6 & 1,4 & 1,2 & 2,1 & 1,4 & 1,1 & 2,7 & 2,0 & 1,8 & 0,083 & 0,088 & 0,270 \\
\hline Teresina & 1,0 & 1,1 & 0,8 & 0,9 & 1,8 & 0,6 & 0,9 & 1,8 & 2,8 & 2,0 & 1,7 & 2,5 & 0,009 & 0,140 & 0,503 \\
\hline Vitória & 1,2 & 0,6 & 1,3 & 1,5 & 1,4 & 1,4 & 1,5 & 1,5 & 1,8 & 2,1 & 1,0 & 1,6 & 0,085 & 0,054 & 0,267 \\
\hline Brasília & 0,6 & 1,0 & 1,3 & 0,9 & 0,3 & 1,7 & 1,9 & 1,6 & 3,1 & 0,7 & 0,8 & 1,4 & 0,342 & 0,063 & 0,090 \\
\hline
\end{tabular}

taxas para os alimentos ultraprocessados e a concessão de subsídios à produção de alimentos saudáveis, como frutas, legumes e hortaliças 16. A taxação de bebidas açucaradas, implementada em 2014 no México, resultou em uma redução de 9,7\% nas vendas até o ano de 201518.

A maior prevalência de obesidade mórbida na faixa etária de 25 a 44 anos pode ser atribuída a fatores sociais e ambientais, tais como independência financeira, estresse no trabalho, tempo reduzido para realização de atividade física e fácil disponibilidade de alimentos ultraprocessados, especialmente para os jovens, tornando-os suscetíveis a hábitos pouco saudáveis 19 .

A maior prevalência da obesidade mórbida no sexo feminino pode estar relacionada a diferenças nos padrões de consumo alimentar, gasto energético e às questões de gênero 10 .

A prevalência de obesidade mórbida mais alta em adultos com baixa escolaridade pode estar relacionada à restrição de informações e ao consumo de alimentos de baixo custo e baixo valor nutricional. Em contrapartida, indivíduos com mais anos de estudo têm maior acesso à informação e aos hábitos de vida mais saudáveis 20 .

Este estudo tem como limitação o fato de os dados serem coletados por entrevistas telefônicas, o que inclui potenciais vieses de informação. 


\section{Conclusão}

A prevalência de obesidade mórbida aumentou em todas as capitais do Brasil, com maiores valores no sexo feminino, faixa etária de 25 a 44 anos, em todos os níveis de escolaridade e regiões do país, constituindo um alerta para a urgente necessidade de adotar medidas que contribuam para sua diminuição. Torna-se necessário, por exemplo, avançar na regulação dos alimentos ultraprocessados e na taxação de bebidas açucaradas, bem como manter o monitoramento dos indicadores do Plano de Enfrentamento das DCNT no Brasil (2011-2022).

\section{Colaboradores}

Todos os autores colaboraram em todas as etapas de produção do artigo.

\section{Informações adicionais}

ORCID: Deborah Carvalho Malta (0000-00028214-5734); Alanna Gomes da Silva (0000-00032587-5658); Luis Antônio Batista Tonaco (00000001-9660-2900); Maria Imaculada de Fátima Freitas (0000-0002-0273-9066); Gustavo VelasquezMelendez (0000-0001-8349-5042).

\section{Referências}

1. World Health Organization. Obesity and overweight. http://www.who.int/en/news-room/ fact-sheets/detail/obesity-and-overweight (acessado em 11/Nov/2018).

2. European Association for the Study of Obesity. By 2035 over 4 million adults will be morbidly obese across England, Wales, and Scotland. EurekAlert! 2018; 25 mai. https://www. eurekalert.org/pub_releases/2018-05/eaftb2o052418.php.

3. Hales CM, Fryar CD, Carroll MD, Freedman DS, Ogden CL. Trends in obesity and severe obesity prevalence in US youth and adults by sex and age, 2007-2008 to 2015-2016. JAMA 2018; 319:1723-5.

4. Finucane MM, Stevens GA, Cowan MJ, Danaei G, Lin JK, Paciorek CJ, et al. National, regional, and global trends in body-mass index since 1980: systematic analysis of health examination surveys and epidemiological studies with 960 country-years and 9.1 million participants. Lancet 2011; 377:557-67.

\section{Agradecimentos}

Os autores do artigo agradecem as equipes técnicas da Secretaria de Vigilância em Saúde do Ministério da Saúde envolvidas na implantação e operacionalização do Vigitel. Financiamento: Ministério da Saúde (Fundo Nacional de Saúde, Convênio no 86).

5. Associação Brasileira para o Estudo da Obesidade e da Síndrome Metabólica. Diretrizes brasileiras de obesidade 2009/2010. 3a Ed. Itapevi: AC Farmacêutica; 2009.

6. Associação Brasileira para o Estudo da Obesidade e da Síndrome Metabólica. Diretrizes brasileiras de obesidade 2016. 4a Ed. http:// www.abeso.org.br/uploads/downloads/92/ 57 fccc403e5da.pdf (acessado em 03/Jan/2019).

7. Sturm R, Hattori A. Morbid obesity rates continue to rise rapidly in the United States. Int J Obes (Lond) 2013; 37:889-91.

8. Lee J, Choi Y-E, Kim D, Lee S, Cho K-H. Trends in socioeconomic costs of morbid obesity among Korean adults, 2009-2013: data from National Health Insurance Service. Obes Res Clin Pract 2018; 12:389-93.

9. Malta DC, Andrade SC, Claro RM, Bernal RTI, Monteiro CA. Trends in prevalence of overweight and obesity in adults in 26 Brazilian state capitals and the Federal District from 2006 to 2012. Rev Bras Epidemiol 2014; 17 Suppl 1:267-76. 
10. Leeners B, Geary N, Tobler PN, Asarian L. Ovarian hormones and obesity. Hum Reprod Update 2017; 23:300-21.

11. World Health Organization. Global Action Plan for the Prevention and Control of NCDs 20132020. http://apps.who.int/iris/bitstream/ handle/10665/94384/9789241506236_eng. pdf? sequence $=1$ (acessado em 18/Nov/2018).

12. Departamento de Análise de Situação de Saúde, Secretaria de Vigilância em Saúde, Ministério da Saúde. Plano de ações estratégicas para o enfrentamento das doenças crônicas não transmissíveis (DCNT) no Brasil 2011-2022. Brasília: Ministério da Saúde; 2011. (Série B. Textos Básicos de Saúde).

13. Ministério da Saúde. Vigitel Brasil 2017: vigilância de fatores de risco e proteção para doenças crônicas por inquérito telefônico: estimativas sobre frequência e distribuição sociodemográfica de fatores de risco e proteção para doenças crônicas nas capitais dos 26 estados. Brasília: Ministério da Saúde; 2018.

14. Bernal RTI, Iser BPM, Malta DC, Claro RM. Sistema de Vigilância de Fatores de Risco e Proteção para Doenças Crônicas por Inquérito Telefônico (Vigitel): mudança na metodologia de ponderação. Epidemiol Serv Saúde 2017; 26:701-12.
15. Iser BPM, Yokota RTC, Sá NNB, Moura L, Malta DC. Prevalência de fatores de risco e proteção para doenças crônicas nas capitais do Brasil - principais resultados do Vigitel 2010. Ciênc Saúde Colet 2012; 17:2343-56.

16. Ministério da Saúde. Guia alimentar para a população brasileira. Brasília: Ministério da Saúde; 2014.

17. Malta DC, Campos MO, Oliveira MM, Iser BPM, Bernal RTI, Claro RM, et al. Prevalência de fatores de risco e proteção para doenças crônicas não transmissíveis em adultos residentes em capitais brasileiras, 2013. Epidemiol Serv Saúde 2015; 24:373-87.

18. The Lancet Diabetes Endocrinology. Sweet success: will sugar taxes improve health? Lancet Diabetes Endocrinol 2017; 5:235.

19. Poobalan A, Aucott L. Obesity among young adults in developing countries: a systematic overview. Curr Obes Rep 2016; 5:2-13.

20. Gorski MT, Roberto CA. Public health policies to encourage healthy eating habits: recent perspectives. J Healthc Leadersh 2015; 7:81-90. 


\section{Abstract}

This study was an assessment of time trends in morbid obesity prevalence in the adult population in Brazil's state capitals from 2006 to 2017. A cross-sectional study was performed with data from the Risk and Protective Factors Surveillance System for Chronic Noncommunicable Diseases Through Telephone Interview (Vigitel), analyzed by simple linear regression. The results showed an upward trend in morbid obesity prevalence in Brazil. Women showed higher prevalence rates (1.3\% in 2006 and $1.9 \%$ in 2017) when compared to men (0.9\% and 1.4\%). The 25-44-year age bracket showed an upward trend from $0.9 \%$ to $2.1 \%(p<0.001)$. There was an increase in morbid obesity in all levels of schooling and all regions of Brazil. The state capitals with upward trends in males were Campo Grande, $\mathrm{Cu}$ ritiba, Fortaleza, Macapá, Manaus, Palmas, Porto Velho, Rio Branco, and Teresina. In females, they were Belo Horizonte, Campo Grande, Rio de Janeiro, and Teresina. The growth in morbid obesity in Brazil sounds a warning on the urgent need to adopt measures to detain it, such as regulation of ultra-processed foods and health education measures for the entire population.

Noncommunicable Diseases; Morbid Obesity; Health Surveys; Health Promotion

\section{Resumen}

Evaluación de la tendencia temporal de la prevalencia de obesidad mórbida en la población adulta de capitales brasileñas entre los años 2006 y 2017. Se realizó un estudio transversal con datos procedentes de la Vigilancia de Factores de Riesgo y Protección para Enfermedades Crónicas No Transmisibles por Entrevista Telefónica (Vigitel), analizados por regresión lineal simple. Los resultados evidenciaron una tendencia de aumento de la obesidad mórbida en Brasil. Las mujeres presentaron mayores prevalencias $(1,3 \%$ en 2006 y 1,9\% en 2017) al compararlas con los hombres $(0,9 \%$ y 1,4\%). En la franja de edad entre 25 a 44 años la tendencia fue de crecimiento de 0,9\% a 2,1\% ( $p<0,001)$. En todos los niveles de escolaridad y regiones de Brasil hubo un aumento de obesidad mórbida. Las capitales que presentaron una tendencia de aumento en el sexo masculino fueron: Campo Grande, Curitiba, Fortaleza, Macapá, Manaus, Palmas, Porto Velho, Río Branco y Teresina. En relación con el sexo femenino: Belo Horizonte, Campo Grande, Río de Janeiro y Teresina. El crecimiento de la obesidad mórbida en el país constituye una alerta para la necesidad urgente de adoptar medidas con el fin de detenerla como pueden ser la regulación de alimentos ultraprocesados, así como acciones educativas para la salud de toda la población.

Enfermedades no Transmisibles; Obesidad Mórbida; Encuestas Epidemiológicas;

Promoción de la Salud
Recebido em 22/Nov/2018

Versão final reapresentada em 25/Fev/2019

Aprovado em 15/Abr/2019 